\title{
Flächensparen zwischen Anspruch und Wirklichkeit. Zur Rolle von Akteuren und Steuerungsinstrumenten in Dorferneuerungsprozessen
}

\author{
Anne Ritzinger ${ }^{1}$ \\ Eingegangen: 1. September 2017 / Angenommen: 7. Juni 2018 / Online publiziert: 7. August 2018 \\ ๑) Springer-Verlag GmbH Deutschland, ein Teil von Springer Nature 2018
}

\section{Zusammenfassung}

Der Beitrag setzt sich am Beispiel des Bayerischen Dorfentwicklungsprogramms mit der Steuerung der Flächenneuinanspruchnahme auf lokaler Ebene auseinander. Hierzu werden mit Methoden der qualitativen empirischen Sozialforschung zwei im Merkmal des Siedlungsdrucks kontrastierende Fallstudien untersucht und die Entscheidungen und Motive beteiligter Akteure betrachtet. Die anhaltend hohe Umwandlung von Freiflächen in Siedlungs- und Verkehrsflächen gilt als eine der großen raumordnerischen und umweltpolitischen Herausforderungen unserer Zeit. Die Reduzierung der Flächeninanspruchnahme stellt ein normatives und strategisches Ziel dar - vor allem in ländlichen Regionen geht die Schere zwischen Flächen- und Einwohnerentwicklung jedoch weiterhin auseinander. Ursächlich sind insbesondere die Entscheidungen lokaler und regionaler Akteure, die unter anderem aufgrund der Konkurrenz um Wohnbevölkerung und Gewerbe und vergleichsweise niedrigeren Bodenpreisen weitere Flächen ausweisen. Im Dorferneuerungsprozess sind vielseitige Ansatzpunkte zur Reduzierung der Flächeninanspruchnahme und Förderung von Innenentwicklung vorhanden. Die Dorferneuerung bietet ein umfangreiches Instrumentenspektrum von der Beteiligung lokaler Akteure und Grundeigentümer über Fachgutachten und Planungskonzepte bis zur Förderung und Umsetzung von Maßnahmen mit Bodenordnung. Dieses Instrumentarium wird jedoch noch nicht systematisch ausgeschöpft. Die kontrastierenden Fallstudien zeigen auf, wie demographische, wirtschaftliche und institutionelle Rahmenbedingungen sich auf die Handlungsspielräume der Akteure und deren Argumentationen auswirken. Flächensparen ist ein Ziel, das zwar politisch und gesellschaftlich erwünscht ist, jedoch im Verhältnis zu anderen Zielsetzungen häufig in der persönlichen, politischen und planerischen Abwägung unterliegt. Abschließend werden Handlungsempfehlungen für die Praxis der Dorferneuerung gegeben und weiterer Forschungsbedarf aufgezeigt.

Schlüsselwörter Akteuranalyse $\cdot$ Bauleitplanung · Dorferneuerung $\cdot$ Flächeninanspruchnahme $\cdot$ Informelle Planung · Ländliche Entwicklung

Dr. Anne Ritzinger

anne.ritzinger@bza.bayern.de

1 Bereich Zentrale Aufgaben, Sachgebiet G3

Dorferneuerung und Integrierte Ländliche Entwicklung,

Bayerische Verwaltung für Ländliche Entwicklung,

Infanteriestraße 1, 80797 München, Deutschland 


\title{
Reducing land take between ambition and reality. The role of actors and instruments in village renewal processes
}

\begin{abstract}
Using the example of the Bavarian Village Renewal Programme, the article investigates which steps and instruments of the village renewal process are capable of steering actors towards a reduction of land take. Two case studies featuring different settlement pressures are analysed using methods of qualitative empirical social research. The continuing transformation of open spaces to built-up areas for settlement and transport is one of the core environmental and planning-related challenges of our time. The reduction of land take is a normative as well as a strategic goal. Particularly in rural areas, however, the divide between land take and population development continues to grow. This phenomenon is the result of decision-making processes of local and regional actors, who are actively zoning out new plots at low prices e.g. in order to achieve a competitive advantage in the intermunicipal struggle for residents and enterprises. Village renewal under the framework of the Federal Land Consolidation Act provides a broad range of instruments, including participation of local actors and landowners, expertise and planning concepts as well as funding and implementation of measures, among others through land readjustment. The article illustrates that while village-renewal processes provide various opportunities to reduce land take and to promote inner development, the objective to reduce land take is not incorporated in the instruments or existing instruments are not fully utilised to that end. The contrasting case studies illustrate how demographic, economic and institutional framework conditions are affecting the actors' scope of action and rational. The aim of reducing land take is an aim that is desirable from a political and societal perspective. Yet when being weighed against other aims these perspectives often succumb to personal or political interests or to perspectives linked to the process of planning. Finally recommendations for the practice of village renewal and an outlook on future needs of research are given.
\end{abstract}

Keywords Actor analysis $\cdot$ Land use planning $\cdot$ Village renewal $\cdot$ Land take $\cdot$ Informal planning $\cdot$ Rural development

\section{Zunehmende Flächeninanspruchnahme als Herausforderung für die Planung: Eine Einleitung}

Die Reduzierung der Flächeninanspruchnahme gilt als eine der großen raumordnerischen und umweltpolitischen Herausforderungen unserer Zeit. Böden und ihre landwirtschaftlichen und ökosystemaren Funktionen sind eine wesentliche Grundlage für die Erhaltung des Lebens auf der Erde (Vereinte Nationen 2013). Boden ist nicht vermehrbar und jeder menschliche Eingriff hat Auswirkungen auf das vielschichtige System Boden. Die deutsche Bundesregierung hat sich bereits vor mehr als zehn Jahren im Rahmen der Nationalen Nachhaltigkeitsstrategie das Ziel gesetzt, bis zum Jahr 2020 die Neuinanspruchnahme von Flächen für Siedlungen und Verkehr auf 30 ha pro Tag zu verringern (Deutsche Bundesregierung 2002: 68) - ein Ziel, das sich aufgrund der anhaltend hohen Umwandlung von landwirtschaftlichen Flächen und anderen Freiflächen in Siedlungsund Verkehrsflächen als illusorisch erwiesen hat. Die Siedlungs- und Verkehrsfläche hat sich von 1992 bis 2015 von 40.305 auf 49.066 Quadratkilometer ausgedehnt und ist damit um 21,7\% angestiegen. ${ }^{1}$ Diese Entwicklung wird angesichts der endlichen Ressource Boden in Wissenschaft und Planungspraxis als nicht zukunftsfähig beurteilt.

\footnotetext{
1 Vgl. https://www.umweltbundesamt.de/node/11184 (28.04.2018).
}

Die Problematik scheint zwar in weiten Kreisen bekannt, dennoch bestehen Umsetzungsdefizite der als notwendig identifizierten Strategien (vgl. Schiller/Gutsche/ Deilmann 2009; Siedentop 2010a). Viele Akteurgruppen sind unterschiedlicher Auffassung, wie prioritär das Thema der Reduzierung der Flächeninanspruchnahme in der Abwägung gegenüber anderen Interessen zu behandeln ist. Auch werden von politischer Seite gegenläufige Entwicklungen unterstützt. Unterstreichen lassen sich diese Beobachtungen durch die Änderung des Baugesetzbuches ${ }^{2}$ im Frühjahr 2017 mit der Einführung des § 13b, der bis Ende 2019 die Ausweisung zusätzlicher Wohnbaugebiete am Siedlungsrand im sogenannten vereinfachten Verfahren ermöglicht. Ein weiteres Beispiel sind die Lockerungen des sogenannten Anbindegebots im bayerischen Landesentwicklungsprogramm, die eine Ansiedlung von Gewerbe und Arbeitsplätzen an nicht integrierten Standorten erleichtern. Kommunale Vertreter von Gemeindeund Landkreistagen sehen darin großteils eine willkommene Stärkung der kommunalen Planungshoheit und erhoffen sich durch die erleichterte Ausweisung von Wohnbauland und Gewerbeflächen unter anderem eine Verbesserung der Kommunalfinanzen (vgl. Bayerischer Gemeindetag 2015; Paul/Taschner 2017). Fach- und Umweltverbände üben hingegen heftige Kritik und befürchten eine starke Zunahme

\footnotetext{
2 Baugesetzbuch (BauGB) in der Fassung der Neubekanntmachung vom 3. November 2017.
} 
der Flächeninanspruchnahme (DASL/Bayerischer Landesverein für Heimatpflege/BUND 2015; Sebald 2015; SRL/ bdla 2017).

Die Ursachen für die weiterhin steigende Flächenneuinanspruchnahme sind auf ein ,komplexes Bündel von demografischen, ökonomischen, raum- und siedlungsstrukturellen Variablen“ (Bock/Preuß 2011: 26) zurückzuführen. Dabei spielen die Nachfrage nach Bauflächen seitens Bevölkerung und Wirtschaft ebenso eine Rolle wie die steigende Wohnfläche pro Person, die Suche nach attraktiven Wohnumgebungen im Grünen sowie teure Mieten und Immobilienpreise. Auch angebotsbezogenen Faktoren scheint eine große Bedeutung für die Zunahme der Flächenneuinanspruchnahme beizumessen zu sein (vgl. BMVBS/BBR 2009). Kommunen und Projektentwickler üben durch Angebotsplanungen Anreize auf die Bauland- und Immobiliennachfrage der privaten Haushalte sowie der Unternehmen aus (vgl. Schiller/Gutsche/Deilmann 2009). Die Angebotspolitik der Gemeinden hat zur Folge, dass Wohnraum unter anderem auch dort geschaffen wird, wo er Prognosen zufolge in Zukunft gar nicht benötigt wird; in prosperierenden Metropolen wird hingegen der Wohnraum knapp und verteuert sich. Eine Studie des Instituts der deutschen Wirtschaft Köln stellt diese gegenläufigen Entwicklungen dar und fordert ein Gegensteuern der Politik (Henger/Schier/ Voigtländer 2015). Auffällig ist die Entkoppelung der Zunahme der Siedlungs- und Verkehrsfläche von der Bevölkerungsentwicklung. Die Siedlungs- und Verkehrsfläche hat im Bundesgebiet im Zeitraum von 1992 bis 2015 um 21,7 \% zugenommen, während die Bevölkerung im selben Zeitraum nur um 1,5\% zugenommen hat. ${ }^{3}$ Diese Entwicklung lässt sich in allen kommunalen Entwicklungstypen beobachten, von stark wachsenden bis hin zu stark schrumpfenden Kommunen (Milbert 2015: 15). Die Entkoppelung ist insbesondere auf eine Erhöhung der Wohnfläche/Kopf von 34,9 Quadratmeter (1991) auf 46,5 Quadratmeter (2014) zurückzuführen, ${ }^{4}$ die ihre Ursache in der fortlaufenden $\mathrm{Ab}$ nahme der durchschnittlichen Haushaltsgröße, der weiterhin zunehmenden Versorgung der Bevölkerung mit Eigenheimen und dem Verbleib älterer Haushalte im Wohneigentum (Remanenzeffekt) hat.

Auch die räumliche Lage der Gemeinden ist von Bedeutung: Je zentraler bzw. dichter besiedelt, desto niedriger ist die Flächeninanspruchnahme je Einwohner (Goetzke/ Schlump/Hoymann et al. 2014: 5). In stagnierenden und schrumpfenden Regionen hingegen wächst die Flächeninanspruchnahme pro Kopf unter anderem durch Abwan-

\footnotetext{
$3 \mathrm{Vgl}$. https://www-genesis.destatis.de/genesis/online?sequenz=tabel leErgebnis\&selectionname $=33111-0006 \&$ regionalschluessel $=$ (02.05.2018).

${ }^{4} \mathrm{Vgl}$. https://www.umweltbundesamt.de/daten/private-haushaltekonsum/wohnen/wohnflaeche\#textpart-3 (20.05.2018).
}

derungsprozesse, aber auch aufgrund angebotsorientierter Bodenpolitik immer weiter. Dies bringt diverse Probleme wie unterausgelastete Infrastrukturen, sinkende Immobilienpreise und damit sinkende Rentabilität von Immobilieninstandhaltung und Nachnutzung mit sich (vgl. Siedentop 2010b).

Die Stellschrauben und Steuerungsmöglichkeiten zur Reduzierung der Flächeninanspruchnahme sind in großen Teilen erforscht und auch von politischer Seite bestehen auf Bundes- und Länderebene Bekenntnisse zur Zielvorstellung Flächensparen (vgl. Möckel 2015). Die tatsächliche Bodenund Flächennutzung wird jedoch fast ausschließlich durch lokale und regionale Entscheidungen bestimmt. Hier scheinen die bisherigen Erkenntnisse nicht anzukommen oder andere Zielsetzungen als prioritär betrachtet zu werden. Es scheint also kein Wissens-, sondern ein Handlungsdefizit bei der Planung und Umsetzung von flächenbezogenen Entscheidungen durch Kommunen zu bestehen.

Das Dorfentwicklungsprogramm mit der Durchführung von Dorferneuerungsverfahren nach dem Flurbereinigungsgesetz (FlurbG) ${ }^{5}$ ist in Bayern ein stark nachgefragtes staatliches Förderprogramm mit einem breiten Spektrum an Handlungsoptionen von der Beteiligung über die Planung bis zur Förderung und flächenwirksamen Umsetzung von Maßnahmen (StMELF 2012: 87). Die Dorferneuerung ist somit ein vielseitiges Instrument der Landentwicklung, das Kommunen, Behörden und insbesondere auch Bürgern und Grundeigentümern umfassende Möglichkeiten eröffnet, ihre lokale Raumentwicklung in eine nachhaltige Richtung zu steuern (vgl. Magel 2014). Der Bedarf nach einer verstärkten Förderung flächensparender Entwicklung und insbesondere der Innenentwicklung ist dabei als Aufgabenschwerpunkt ins Bewusstsein von Politik und Verwaltung gerückt (vgl. StMLF 2006; Bayerische Verwaltung für Ländliche Entwicklung 2014).

Vorliegender Beitrag beruht auf den Erkenntnissen einer Dissertation, die mit qualitativen Methoden die Rolle der Akteure im Dorferneuerungsprozess untersucht (Ritzinger 2016). Das Erkenntnisinteresse bezieht sich insbesondere darauf, mögliche Ursachen für das Handeln der Akteure für eine Flächeninanspruchnahme nachzuvollziehen und mit den im Dorferneuerungsprozess angewandten Steuerungsinstrumenten in Beziehung zu setzen. Die zentrale Fragestellung der Arbeit lautet: Welche Ansatzpunkte bietet der Dorferneuerungsprozess des Bayerischen Dorfentwicklungsprogramms, um die lokalen Akteure in Richtung einer Reduzierung der Flächeninanspruchnahme $\mathrm{zu}$ steuern?

\footnotetext{
5 Flurbereinigungsgesetz in der Fassung der Bekanntmachung vom 16. März 1976, das zuletzt durch Artikel 17 des Gesetzes vom 19. Dezember 2008 geändert worden ist.
} 
Der Beitrag gibt einen kurzen Überblick zum Forschungsstand im Themenfeld, stellt das methodische Vorgehen und die Operationalisierung der theoretischen Ansätze für die Untersuchung dar und geht auf ausgewählte Ergebnisse und mögliche Schlussfolgerungen für Wissenschaft und Praxis ein. Im Folgenden werden alle baulichen Entwicklungen einbezogen, die Grund und Boden beanspruchen, zunächst unabhängig davon, wie diese planungsrechtlich einzuordnen sind und ob eine tatsächliche Versiegelung der Flächen stattgefunden hat. Denn auch durch die planungsrechtliche „Inanspruchnahme“ wird die Fläche in der Regel dauerhaft oder für lange Zeiträume anderen Nutzungen entzogen. Bestrebungen zur Reduzierung der Flächeninanspruchnahme können auf verschiedene Teilziele heruntergebrochen und operationalisiert werden, beispielsweise durch Projekte zur Förderung der Innenentwicklung zur Vermeidung einer weiteren Außenentwicklung und Zersiedelung.

\section{Dorfentwicklung und Steuerung der Flächeninanspruchnahme}

Dorferneuerungen bzw. Dorfentwicklungsmaßnahmen werden in jedem Bundesland und in den europäischen Nachbarländern unterschiedlich durchgeführt und verfügen jeweils über andere Regelungen, Instrumente und Akteurbezüge. In Deutschland erfolgt die programmatische Unterstützung der Entwicklung und Sanierung von Dörfern entweder durch agrarstrukturelle oder städtebauliche Programme (vgl. Magel 1991; Dehne 2010; Henkel 2012; Kötter/Voß 2012). In den meisten Bundesländern wurde eine klare Aufgabenteilung zwischen den zuständigen Ressorts vereinbart, die sich beispielsweise an der Einwohnerzahl der Gemeinden orientiert. Nur in wenigen Bundesländern gibt es eigenständige Landesprogramme für Dorfentwicklung oder Dorferneuerung wie in Bayern. Hier werden Dorferneuerungen als Verfahren nach dem Flurbereinigungsgesetz durch die Verwaltung für Ländliche Entwicklung durchgeführt, eine Teilnehmergemeinschaft zur Planung und Umsetzung von Maßnahmen eingerichtet und umfassende Planungs- und Beteiligungsprozesse angestoßen (vgl. Hanns-Seidel-Stiftung 1989). Einen ähnlichen Aufbau der Dorferneuerung haben die Bundesländer Thüringen und Sachsen. Eine Dorferneuerung nach dem Flurbereinigungsgesetz verfolgt den umfassenden Auftrag der Förderung der Landentwicklung und der Verbesserung der Lebens- und Arbeitsbedingungen in den Dörfern (vgl. Spangenberger 2008). Zielvorgaben in Richtung einer nachhaltigen Raumentwicklung, die auch für die Landentwicklung und Dorferneuerung gültig sind, ergeben sich unter anderem durch die Vorgaben der Raumordnung und Landesplanung (z. B. das Bayerische Landesentwicklungsprogramm; Bayerische Staatsre- gierung 2013), das Flurbereinigungsgesetz und die Bayerischen Dorferneuerungsrichtlinien. Hier wird beispielsweise als Zuwendungszweck benannt: „Durch die Dorferneuerung sollen [...] die Innenentwicklung der Dörfer und der sparsame Umgang mit Grund und Boden gefördert, der eigenständige Charakter ländlicher Siedlungen und die Kulturlandschaft erhalten [...] werden“" (StMELF 2015: 6).

Das Thema Innenentwicklung und Reduzierung der Flächeninanspruchnahme spielt in den Dorfentwicklungsmaßnahmen der Länder eine zunehmende Rolle (vgl. für Hessen Boos-Krüger 2009; vergleichend zur Rolle der Innenentwicklung in Hessen, Mecklenburg-Vorpommern, Niedersachsen, Nordrhein-Westfalen und Schleswig-Holstein Peter/Fengler/Moser 2013; für Niedersachsen Grube 2006; Voß/Güldenberg/Kirsch-Stracke et al. 2011; Steffenhagen/ Weitkamp 2015; für Baden-Württemberg Malburg-Graf/ Schmettow 2012; für Brandenburg Kötter/Langer 2014; für Bayern Rill/Drago/Groß 2014; Lintzmeyer/Schwarz/ Müller-Herbers et al. 2015).

In Bezug auf die Steuerung der Flächenneuinanspruchnahme sind bereits umfassende Untersuchungen in verschiedenen Disziplinen getätigt worden (vgl. Schmalholz 2005; Job/Pütz 2006; BBR 2007; Klemme 2009; Kötter/ Weigt/Frielinghaus et al. 2009; Bock/Hinzen/Libbe 2011; Weith/Besendörfer/Gaasch et al. 2013). Zentrale Erkenntnis der wissenschaftlichen Diskussion ist derzeit, dass multiinstrumentelle Ansätze, also Kombinationen von Instrumenten mit unterschiedlichen Steuerungsmethoden, notwendig sind, um das Ziel „Reduzierung der Flächeninanspruchnahme" in seiner thematischen Breite und flächenhaft umzusetzen und die letztlich über die Bodennutzung entscheidenden Akteure wie Kommunalpolitiker und Grundeigentümer zu erreichen (vgl. Möckel 2015).

\section{Methodisches Vorgehen und kurze Vorstellung der Fallstudien}

Der für die Untersuchung gewählte, qualitative Forschungsansatz ist darauf gerichtet, das Handeln der Akteure in seinen kontextuellen Bedingungen, spezifischen Ausprägungen und Konsequenzen zu erfassen (vgl. Blatter/Janning/ Wagemann 2007).

Es wurden zwei Fallstudien verwendet, um in explorativer und kontrastierender Weise mögliche Ansatzpunkte für die Reduzierung der Flächeninanspruchnahme im Dorferneuerungsprozess zu eruieren. Die Fallstudien kontrastieren in ihren Rahmenbedingungen. Charakteristisch für die empirische Arbeit mit Fallstudien ist das Ziel, den untersuchten Fall ganzheitlich zu erfassen. Daher wird, insbesondere in fallzentrierten Fallstudiendesigns, angestrebt, möglichst alle für das Untersuchungsobjekt relevanten Dimensionen in die Analyse einzubeziehen (George/Bennett 2005: 207). 
Verschiedene Vorannahmen führten schrittweise zu einer Eingrenzung der möglichen Dorferneuerungsprozesse, die für die Fallstudien in Frage kamen. Als Kriterien zur Auswahl der Fallstudien dienten unter anderem die unterschiedliche Lage im Raum (Bayern; unterschiedliche Zuständigkeiten der Ämter für Ländliche Entwicklung; unterschiedliche Bevölkerungs- und Immobilienpreisentwicklung), der Verfahrenstyp der Dorferneuerung (Umfassendes Verfahren nach $\S \S 1,4,37$ FlurbG $^{6}$ ) und die Setzung des Themas Siedlungsentwicklung als eines der Schwerpunktthemen in den Verfahren.

Um in den Fallstudien vergleichbare Untersuchungsstrukturen aufzubauen und die Fragestellung sowohl auf der prozessualen Ebene sowie aus der Akteurperspektive zu beantworten, wurden die Methoden der Prozess- und der Akteuranalyse ausgewählt. Neben der zeitlichen Rekonstruktion des Prozessablaufs stand in der Prozessanalyse die Untersuchung des Zusammenwirkens von Einflussfaktoren im Mittelpunkt der Betrachtung. Es wurde insbesondere auf die Auswertung von Verfahrens- und Planungsunterlagen sowie Protokollen zurückgegriffen. Die methodischen Schritte zur Durchführung der Akteuranalysen in den Fallstudien erfolgten nach Hirsch Hadorn und Wölfing Kast (2002) sowie GTZ (o.J.) und Schwing/Fryszer (2015). Es wurden qualitative leitfadengestützte Interviews mit 30 Personen geführt. Die Gesprächspartner waren beispielsweise Bürgermeister, Mitglieder des Gemeinderates und des Vorstands der Teilnehmergemeinschaft der Dorferneuerung sowie Planer, Mitarbeiter des Amtes für Ländliche Entwicklung, Anwohner und Investoren. Die Auswertung der Interviews erfolgte mittels thematischer Kodierung (vgl. Meuser/Nagel 2005: $80 \mathrm{ff}$.). Daraufhin wurden Gemeinsamkeiten und Unterschiede zwischen den Akteuren und zwischen den Fällen herausgearbeitet (vgl. Lamnek 1995: $36 \mathrm{ff}$. .).

Als Fallstudien dienten die Dorferneuerungsprozesse in der Gemeinde Weyarn im Landkreis Miesbach, Oberbayern und im Ortsteil Obbach der Gemeinde Euerbach, Landkreis Schweinfurt, Unterfranken. Beide Gemeinden sind für ihre guten Ansätze nachhaltiger Siedlungsentwicklung bekannt. Weyarn nahm beispielsweise als Untersuchungsgemeinde am Forschungsprojekt ,21 Gemeinden - Gute Beispiele für eine nachhaltige Kommunalentwicklung" des Bayerischen Umweltministeriums mit Qualitätszirkeln zum Thema Siedlung/Boden/Fläche teil (vgl. Magel/Franke 2007) und wurde 2004 mit dem Europäischen Dorferneuerungspreis ausgezeichnet. Die Gemeinde Euerbach mit dem Ortsteil Obbach diente unter anderem bereits als Fallstudie für das Modellprojekt „FLIZ - Flächenmanagement in interkommuna-

\footnotetext{
${ }^{6}$ Flurbereinigungsgesetz in der Fassung der Bekanntmachung vom 16. März 1976, das zuletzt durch Artikel 17 des Gesetzes vom 19. Dezember 2008 geändert worden ist.
}

ler Zusammenarbeit“ (vgl. LfU 2009) und bekennt sich in der „Oerlenbacher Erklärung“ zur Innenentwicklung und zum Flächensparen in der Interkommunalen Allianz Oberes Werntal (vgl. Schmied 2009). Während Obbach durch Stagnation gekennzeichnet war, verzeichnete Weyarn ein gemäßigtes, aber kontinuierliches Bevölkerungswachstum, insbesondere durch die Lage im Großraum München. In Obbach bestanden ein zunehmendes Leerstandsrisiko und ein stärkeres Bewusstsein für die Notwendigkeit von Innenentwicklung. In Weyarn herrschte zwar eine hohe Wertschätzung für die Erhaltung des Ortsbildes, aufgrund der attraktiven Lage und des Zuwanderungsdrucks bestand jedoch eine starke Nachfrage nach Wohnbauflächen durch Einheimische und Zuziehende, die die Gemeinde auch zu befriedigen versuchte.

In der Prozess- und Akteuranalyse wurden im Dorferneuerungsprozess stattfindende Ereignisse, die in Zusammenhang mit der Siedlungsentwicklung stehen, abgebildet. Für die detaillierte Akteuranalyse wurden in jeder Fallstudie zwei raumrelevante Entscheidungen ausgewählt, die im Dorferneuerungsprozess eine Rolle spielten und bei denen ein Großteil der lokalen Akteure eingebunden war. In Weyarn waren dies die Wohnbebauung des Klosterangers und die Ansiedlung eines großflächigen Gewerbebetriebes, in Obbach das Modellprojekt Bauhütte und Sanierung des Nebengebäudes der Bauhütte sowie die Neubebauung am Ortsrand für einen privaten Investor. An diesen Bebauungsentscheidungen wurden die Akteurskonstellationen und Handlungslogiken der Akteure intensiver betrachtet (vgl. Ritzinger 2016: 83 ff.). In Kapitel 5 wird auf die Bebauungsentscheidungen exemplarisch eingegangen.

\section{Interpretationsansätze des Handelns von Akteuren und dessen Steuerung}

Um sich dem Thema Steuerung der Flächenneuinanspruchnahme zu nähern, scheint es erforderlich, sich mit dem Handeln von Akteuren und den Möglichkeiten einer Steuerung der räumlichen Entwicklung durch Akteure auseinanderzusetzen. In der sozialwissenschaftlichen Theoriebildung stellen Akteur- oder Handlungstheorien auf der einen und systemorientierte Theorien auf der anderen Seite die großen paradigmatischen Fundierungen dar. In den vergangenen Jahren fanden insbesondere akteurtheoretische Ansätze verstärkt Verwendung (vgl. Klemme 2009; Dosch 2010; Diller 2013). Eine Ursache scheint darin zu liegen, dass eine systemtheoretische Betrachtung von Gesellschaft in Hinblick auf die Steuerung nachhaltiger Raumentwicklung die Einflüsse der Akteure nicht ausreichend differenzieren kann. Auch an Forschungen, die am GovernanceKonzept orientiert sind, wird ähnliche Kritik geübt (vgl. Mayntz 2005; Fürst 2015). Handlungstheorien seien sys- 
temorientierten Ansätzen gegenüber insbesondere bei der Erforschung von Verhandlungssystemen, konkreten Handlungsmöglichkeiten sowie Prozessdynamiken im Vorteil, da sie viel stärker auf die Austauschprozesse zwischen Akteuren fokussieren statt auf gesellschaftsweite Entwicklungen und akteurübergreifende Trends (Braun 2000: 172f.).

Im Folgenden werden Erklärungsansätze aus der soziologischen und politikwissenschaftlichen Akteur- und Governance-Forschung (Scharpf 2006) mit politik- und planungswissenschaftlichen Ansätzen der Prozessforschung (Selle 2005; Blatter/Janning/Wagemann 2007; Fürst/Scholles 2008) verbunden, um die Handlungen der Akteure im Dorferneuerungsprozess, deren Auswirkungen auf die Inanspruchnahme von Flächen und die Verwendung möglicher Steuerungsansätze einzuordnen. Raumwirksames Handeln und politische Entscheidungen sind in der Regel eingebettet in einen Entscheidungsprozess mit mehreren Akteuren in unterschiedlichen institutionellen Rahmenbedingungen. Nach Scharpf (2006: 87) wird das Handeln der Akteure nicht allein durch ihre institutionelle Umwelt, ihre Präferenzen und Wahrnehmungen sowie ihre Handlungsressourcen ermöglicht und begrenzt, sondern vor allem durch das Handeln anderer Akteure, sogenannte Akteurkonstellationen. Wer Planungsprozesse $\mathrm{zu}$ gestalten und $\mathrm{zu}$ steuern hat, muss sich intensiv damit auseinandersetzen, wer die relevanten Akteure sind, welche Interessen sie verfolgen und mit welchen Mitteln sie diese durchsetzen können, also welche Strategieoptionen sie haben (Fürst 2010a: 20). Bei der Analyse sollten jedoch nicht nur diejenigen Akteure berücksichtigt werden, ,die sichtbar und bewusst ein System beeinflussen" (Ratter 2006: 117), sondern auch solche, die zunächst nicht als Entscheidungsträger auffallen.

Verschiedene Akteure mit unterschiedlichen Handlungsorientierungen und Einflusspotenzialen auf lokaler und überörtlicher Ebene nehmen Einfluss auf die Fläche und versuchen, die Flächennutzung mit unterschiedlichen Steuerungsinstrumenten $\mathrm{zu}$ beeinflussen. Vor Ort stoßen diese Ziele jedoch auf eine Vielzahl teils widersprüchlicher Interessen von Akteuren, die eine formalistische Durchsetzung am Schreibtisch erstellter Planungen unmöglich machen (vgl. Wiechmann 2014). Insbesondere seit die Forderung einer nachhaltigen Entwicklung auf der politischen Agenda steht, ist die Frage nach der Aushandlung von Interessen und der Steuerbarkeit von räumlichen Entwicklungen zentral.

Die flächenbezogenen Auswirkungen des Handelns sind den Akteuren teilweise bewusst, teilweise spielen sie eine untergeordnete Rolle oder werden ausgeblendet (vgl. Einig 2010: 167). Auf Bundes- und Länderebene ist die Akteurlandschaft aufgrund der institutionellen Fragmentierung sehr heterogen. Nur wenige Akteure, die bodenrelevante Entscheidungen verantworten, nehmen dies auch aktiv als Gestaltungsauftrag wahr. Sie sehen sich selbst nicht als „Bodenakteure“, sondern haben ihr primäres Thema, z. B. die Landwirtschaft oder die Umwelt, im Fokus (vgl. Jürges 2016). Diese Vorgaben wirken bis auf die lokale Ebene und auch hier, in der Kommune, nehmen viele Akteure Einfluss auf die Nutzung von Grund und Boden. Die Rollen der Akteure können als Grundstückseigentümer, Grundstücksnachfrager, Planer, Investor oder beispielsweise Umweltverbände sehr unterschiedlich und durch gegensätzliche Interessen geprägt sein (vgl. Dosch 2010: 207f.).

Akteure unterliegen vielfältigen Anreizsystemen, die ihr Handeln für oder gegen Flächeninanspruchnahme beeinflussen. Diese lassen sich verschiedenen Interaktionsformen zuordnen, die Akteuren zur Verfügung stehen (Scharpf 2006: 90f.). Insbesondere finanzielle Mechanismen positiver Art (wie Anreize unter anderem durch Förderprogramme, spezielle Einnahmemöglichkeiten) oder negativer Art (wie Strafzahlungen oder hohe Kosten) wirken steuernd. Zudem nehmen Rahmenbedingungen wie gesetzliche Standards und rechtsverbindliche Vorschriften als sanktionierbare Vorgaben Einfluss. Auf der Basis unterschiedlicher Ansätze (vgl. Selle 2005: 120; Fürst 2010b: 183) wurden in der empirischen Untersuchung sechs Kategorien der Steuerung des Handelns von Akteuren verwendet:

- Regulativ-hoheitliche Instrumente

- Planerisch-informelle Instrumente

- Instrumente der Information, Überzeugung und Kommunikation

- Finanzierungs- und Förderinstrumente

- Instrumente der Marktteilnahme

- Instrumente der Prozesssteuerung und Organisationsentwicklung

\section{Darstellung ausgewählter Ergebnisse und Diskussion}

Im Folgenden werden auf Basis von Ritzinger (2016) ausgewählte Ergebnisse bezüglich der Rolle der Akteure und der Steuerungsmöglichkeiten in der Dorferneuerung dargestellt.

\subsection{Handlungslogiken der Akteure}

Das Handeln der Akteure in Bezug auf Flächeninanspruchnahme wurde in ausgewählten Bebauungsentscheidungen intensiver betrachtet (vgl. Ritzinger 2016: 83 ff.). In Weyarn wurde unter anderem die Wohnbebauung des Klosterangers untersucht, einer 40.000 Quadratmeter großen Fläche am Ortseingang mit ortsbildprägendem Blick zum Kloster. Im Leitbild der Dorferneuerung war zu Beginn der 1990er-Jahre festgehalten worden, dass diese Wiese unbebaut bleiben soll und auch im Dorferneuerungsplan und im gemeind- 
lichen Flächennutzungsplan wurde diese Zielsetzung festgehalten. 2009 wurde im Zusammenhang mit der Vitalisierung des Ortskerns eine Wohnbebauung angedacht, was bei einigen Akteuren, insbesondere Anwohnern, für Unmut sorgte. Ein Bürgerentscheid führte letztlich zur Bebauung des Klosterangers. Aufgrund der Größe der Fläche handelt es sich dabei baurechtlich nicht um Innenentwicklung im engeren Sinne, sondern um einen Außenbereich im Innenbereich, der an bestehende Bebauung angebunden ist. In der Fallstudie Obbach wurde unter anderem das Modellprojekt Bauhütte als Ansatz zur Förderung der Innenentwicklung untersucht. Für die „Bauhütte Obbach“ wurde im Ortskern ein leerstehendes Gebäude in baufälligem $\mathrm{Zu}$ stand von der Teilnehmergemeinschaft der Dorferneuerung aufgekauft. Ziel war es zu veranschaulichen, wie eine Sanierung im Altort aussehen kann und so andere Einwohner zur Innenentwicklung zu motivieren. Ebenso konnten die Grundstücksgrenzen und Durchfahrtsrechte durch Bodenordnung optimiert und ein moderner Bau als Informationsgebäude errichtet werden. Ein Großteil der Bürgerschaft steht dem Projekt jedoch eher indifferent gegenüber.

Die systematische Betrachtung der Akteurkonstellationen verdeutlichte die Veränderung der Einstellungen der Akteure. In drei von vier Beispielen wandelte sich die Einstellung der Kernakteure (wie Bürgermeister, Gemeinderat, Vorstand der Teilnehmergemeinschaft) zur Realisierung der Bebauung von „Dagegen“ zu „Dafür“" oder „Egal“. Es zeigte sich, dass die Reduzierung der Flächeninanspruchnahme keine Chance auf Verwirklichung hat, wenn sie im tatsächlichen oder vermuteten Widerspruch mit individuellen Interessen oder demographischen und wirtschaftlichen Zielsetzungen steht.

Die Fallstudien legen eine Interpretation von Handlungen im Sinne des „Entscheidens als soziale Praxis“ (Wilz 2010: 128) nahe: Unterschiedliche objektive und subjektive Aspekte führen in der Regel zur Entscheidung. Neben den fachlichen Anforderungen und materiellen Rahmenbedingungen spielten subjektive Faktoren wie persönliche Betroffenheit oder entscheidungsrelevante Kontakte eine Rolle. Das Einflusspotenzial der Akteure auf den Dorferneuerungsprozess und auf flächenbezogene Entscheidungen wird zum großen Teil durch ihre formale Position und die damit verbundenen Mitsprache- oder Entscheidungsrechte bestimmt. Insbesondere der Gemeinderat, der Vorstand der Teilnehmergemeinschaft und das Amt für Ländliche Entwicklung haben zentrale Machtpositionen in der Dorferneuerung. Doch auch Akteure, die mit wenig formaler Macht ausgestattet sind, haben die Möglichkeit, durch ihre Rolleninterpretation starken Einfluss auf den Prozessverlauf zu nehmen. Hier sind insbesondere der Planer und die sich in Arbeitskreisen engagierenden Bürger zu nennen. Sie können durch ihre Überlegungen und fachlichen Vorschläge wesentliche Impulse im Prozess setzen, wie sich auch in den beiden Fallstudien zeigt. Je stärker die Organisationsform, z. B. durch den Zusammenschluss als Bürgerinitiative in Weyarn, desto größer ist die Eventualität, sich in die Entscheidungen im Prozess einzubringen. Maßgeblichen Einfluss - auch ohne formale Einbindung in den Dorferneuerungsprozess - nehmen häufig privatwirtschaftliche Akteure durch ihre Investitionsinteressen. Letztlich wurden die baulichen Entscheidungen jedoch stets durch die Gemeinderäte getroffen. Die Akteure der Dorferneuerung hatten in beiden Fallstudien in der Regel eine beratende Rolle, jedoch keine Vetomacht. Nur im Beispiel Bauhütte Obbach waren die Akteure der Dorferneuerung durch den Erwerb des Grundstücks und die Finanzierung direkt involviert.

In der empirischen Untersuchung wurde überprüft, inwiefern das übergeordnete Ziel der Reduzierung der Flächeninanspruchnahme auch in den ortsbezogenen Zielsetzungen und in der Wahrnehmung und dem Handeln der Akteure eine Rolle spielt. Eine kritische Grundhaltung gegenüber einem weiteren baulichen Wachstum der Gemeinde zeigte sich bei den Kernakteuren in beiden Fallstudien, diese ist allerdings vor dem Hintergrund der unterschiedlichen Rahmenbedingungen zu interpretieren: In Weyarn präferieren die Akteure ein geringes Wachstum und die Ansiedelung vor allem einheimischer Bevölkerung und begründen dies mit dem hohen Siedlungsdruck, der zum Schutz des Ortsbildes zu kanalisieren sei. In Obbach förderte die hohe zunehmende Zahl leerstehender Gebäude die Akzeptanz für die Notwendigkeit der Innenentwicklung. Die Reduzierung der Flächeninanspruchnahme an sich war jedoch in beiden Fällen kein explizites Ziel der Akteure.

Die Analyse von Rollenüberschneidungen zeigte in beiden Fallstudien ein komplexes Akteurgeflecht. Insbesondere die gleichzeitige Rolle einer Person als Vorsitzender des Vorstands der Teilnehmergemeinschaft und als Mitarbeiter des Amts für Ländliche Entwicklung ist interessant. Dieser Akteur hat sowohl eine machtvolle als auch eine mit inneren Widersprüchen behaftete Position. Er soll einerseits neutral den Verwaltungsprozess begleiten, sich fachlich kompetent im Sinne der Verwaltungsvorgaben einbringen und gleichzeitig den Erwartungen vor Ort entsprechend viele Projekte und deren Förderung ermöglichen. In den Fallstudien artikulierten die Befragten diese Widersprüche, zogen sich jedoch in der Regel in Bezug auf Themen der Flächenneuinanspruchnahme unter anderem aufgrund der Aufgabenbelastung auf eine neutrale Position zurück. In den untersuchten Dorferneuerungsprozessen werden von einigen Befragten Konflikte zwischen Privatinteressen und Gemeinwohl sehr differenziert diskutiert. Im Beispiel des Klosterangers in der Fallstudie Weyarn wurden Interessen des Gemeinwohls sowohl von den Befürwortern als auch von den Gegnern der Bebauung herangezogen. Den Gegnern der Bebauung wird von den anderen Akteuren ein NIM- 
BY-Verhalten ${ }^{7}$ unterstellt (vgl. Menzl 2014). Die Anwohner wollten verhindern, dass vor ihrem Grundstück weitere Bebauung entsteht und damit anderen das nicht gönnen, was sie selbst in Anspruch nehmen durften: den Bau eines Eigenheims. Die Gegner der Bebauung beriefen sich zu ihrer Legitimation auf viele Aspekte, die eher dem Gemeinwohl zuzuordnen sind: Die Wahrung der einmaligen Ansicht des Ortes und der wertvollen Kulturlandschaft und die Verantwortung, den nachfolgenden Generationen eine dörfliche Heimat zu erhalten, erforderten es, eine dauerhafte Bebauung der Fläche zu verhindern. Die Gegner der Bebauung übernehmen damit viele gemeinwohlbezogene Argumente, die zu Beginn des Prozesses von Seiten des Gemeinderats und der Dorferneuerungsakteure vertreten wurden.

In diesem Spannungsfeld eine objektive Wahrheit und den Grad der jeweiligen Partikularinteressen zu ermitteln, ist nicht möglich. Aushandlungsprozesse für die Umsetzung planerischer Entscheidungen verlaufen in demokratischen Gesellschaften zumeist so, dass sich die Interessen der Mehrheit durchsetzen. Die Beteiligungs- und Diskussionsformen der Dorferneuerung können ein geeignetes Mittel sein, um unterschiedlichen Interessen zur Sichtbarkeit zu verhelfen und einen Weg in die Abwägung zu eröffnen. Finden Belange des Flächensparens und der Erhaltung der Landschaft keine Berücksichtigung oder Fürsprache, so ist dies Aufgabe der öffentlichen Akteure, darauf aufmerksam zu machen. In der Fallstudie sahen die Gegner der Bebauung in den Diskussionsformen der Dorferneuerung keine Möglichkeit, ihre Interessen durchzusetzen und wählten als letztes Mittel den Bürgerentscheid. Letztendlich fand damit eine Abstimmung unter Einbezug aller Wahlberechtigten über die Frage der Flächeninanspruchnahme statt, die zugunsten einer Bebauung ausfiel.

\subsection{Steuerungsinstrumente im Dorferneuerungsprozess}

Im Dorferneuerungsprozess kommen Verfahrensschritte nach dem Flurbereinigungsgesetz zur Anwendung, wodurch Möglichkeiten der Beteiligung von Bürgern, Grundeigentümern, Gemeinde und Trägern öffentlicher Belange sowie zur informellen Dorferneuerungsplanung und zur Bodenordnung nach dem Flurbereinigungsgesetz bestehen. Diese Instrumente der Planung, Beteiligung und Bodenordnung lassen sich, bezugnehmend auf die Überlegungen zu Instrumententypen, zur Steuerung räumlicher Entwicklungen in die abgeleiteten Kategorien einordnen (vgl. Tabelle 1). Durch den institutionellen Kontext des „Amts für Ländliche Entwicklung" als hierarchische Organisation verfügt dieses über ein breites Spektrum von Interakti-

$\overline{7 \text { Nimby steht }}$ als englischsprachiges Akronym für „Not In My Back Yard".

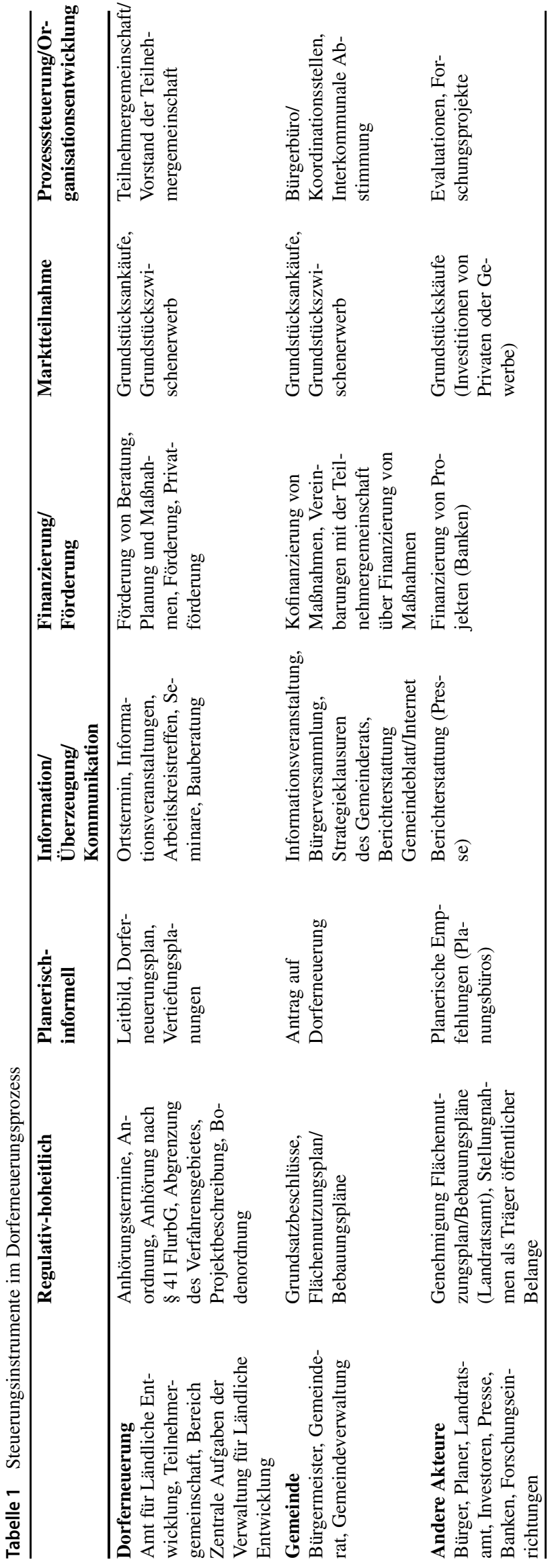


onsformen. Der Prozess ist durch gesetzliche und verwaltungsrechtliche Vorgaben strukturiert, in vielen Aspekten jedoch auch flexibel für Veränderungen durch die Akteure und den Einsatz weiterer Steuerungsinstrumente. Als erfolgversprechende Ansatzpunkte für eine Reduzierung der Flächenneuinanspruchnahme erwiesen sich Instrumente aus unterschiedlichen Kategorien. Aus den Fallstudien lassen sich dafür unter anderem die flächendeckende Erfassung der Innenentwicklungspotenziale in Obbach, die umfassende Bürgerbeteiligung in Weyarn und die aktive Bodenpolitik durch den Grundstückszwischenerwerb der Teilnehmergemeinschaft in Obbach bzw. die Bodenbevorratung der Gemeinde in Weyarn hervorheben.

Ansatzpunkte für eine Reduzierung der Flächeninanspruchnahme im Dorferneuerungsprozess bieten sich durch die Interaktionen von Akteuren in Akteurkonstellationen und können durch Steuerungsinstrumente beeinflusst werden. Insbesondere die Mitarbeiter der Verwaltung für Ländliche Entwicklung entscheiden über den Einsatz von Instrumenten und damit auch über die Einbindung weiterer Akteure und Akteurkonstellationen, in denen raumbezogene Entscheidungen stattfinden. Zwar sind die handlungsleitenden Motive und Interessen der Personen nur zu einem Teil durch die Steuerungsinstrumente beeinflussbar. Dennoch bieten sich mit den Steuerungsinstrumenten - insbesondere aus den Bereichen informelle Planungsmethoden, informatorische Instrumente und Prozessgestaltung - viele Ansatzpunkte, um Motive und Interessen zu erfassen, diese zu diskutieren, abzuwägen und auch zu verändern - mehr als bisher genutzt.

Innenentwicklungsstrategien scheitern oftmals an der mangelnden Verkaufsbereitschaft und den überhöhten Preisvorstellungen der Eigentümer für ihre Immobilien. Ihr Eigeninteresse steht also letztlich einer effizienten Siedlungsstruktur und Belebung des Ortes durch die Wiedernutzung von Leerständen entgegen. Um diese Flächen für die Innenentwicklung zu aktivieren, bietet sich beispielsweise eine Reform der Grundsteuer im Sinne einer Besteuerung am Verkehrswert an. Dadurch würde eine langfristige private Bodenbevorratung finanziell wesentlich unattraktiver (vgl. Jering/Lindemann/Seidel et al. 2003; Ulmer/Renn/Ruther-Mehlis et al. 2007). Darüber hinaus müssen ökonomische Anreize oder Kompensationsmechanismen geschaffen werden, um eine weitgehende Kostenparität zwischen dem Neubau einerseits und der Sanierung des Altbaus andererseits herzustellen. Solche Förderungen oder Ausgleichszahlungen könnten im Rahmen des Dorferneuerungsprozesses entwickelt und aus verschiedenen Fördertöpfen gespeist werden.

Auch die interkommunale Abstimmung von Baulandausweisungen ist sowohl in wachsenden als auch in schrumpfenden Regionen von Bedeutung für eine nachhaltige Siedlungsentwicklung. Wenn eine Innenentwicklungsstra- tegie wirklich greifen soll, so müssen sich benachbarte Gemeinden darauf verlassen können, dass die jeweils andere keine Neubaugebiete ausweist und damit potenzielle Bauherren abzieht. Hier sind gemeinsame informelle Planungen wie interkommunale Allianzen (z. B. im Oberen Werntal) und Integrierte Ländliche Entwicklungskonzepte (ILEK) ein erster Schritt, sowohl eine umfassende Analyse der Siedlungsentwicklung zu ermöglichen wie auch gemeinsame informatorische Grundlagen zu schaffen. Weiterführend können bodenpolitische Selbstverpflichtungen oder Kompensationsfonds sein.

Diese Beispiele zeigen, dass im Rahmen der Dorferneuerung Möglichkeiten bestehen, von allen Akteuren anerkannte Verfahren der Entscheidungsfindung und Konfliktlösung über knappe Ressourcen zu etablieren. Der Dorferneuerungsprozess kann als institutioneller Rahmen zur Abstimmung der Logiken der Akteure unter besonderer Berücksichtigung von Gemeinwohlinteressen ohne eigene Lobby gesehen werden. Für eine stärkere Verankerung des Themas im Prozess könnte die Reduzierung der Flächeninanspruchnahme als Querschnittsziel behandelt und bei allen Maßnahmen zur Abklärung in der Abwägung anstehen. Hier sollte das Thema als ein gleichwertiges Ziel von den Vertretern der öffentlichen Hand eingespeist werden, um anhand klarer Regeln der Entscheidungsfindung gewürdigt $\mathrm{zu}$ werden.

\section{Fazit und Schlussfolgerungen für Praxis und Wissenschaft}

Flächensparen scheint ein Thema zu sein, das zwar politisch und gesellschaftlich erwünscht ist, jedoch im Verhältnis zu anderen Zielsetzungen häufig in der persönlichen, politischen und planerischen Abwägung unterliegt. Eine Reduzierung der Flächeninanspruchnahme liegt zwar gesamtgesellschaftlich und aus ökologischer Perspektive im Interesse des Gemeinwohls, bietet jedoch meist keinen für die Einzelperson direkt erfahrbaren Nutzen. Eine Bebauung der Fläche liegt, wie die Fallstudien belegen, zumeist im individuellen Interesse des Grundeigentümers bzw. Bauherren oder der kommunalen Entscheidungsträger. Die Vorteile einer Nichtbebauung wie beispielsweise Erhaltung des Landschafts- und Ortsbildes oder Verbesserung des Wasserrückhalts sind hingegen nicht unmittelbar monetarisierbar (vgl. Naturkapital Deutschland 2012).

Wissenschaftliche Untersuchungen sollten im Sinne eines transdisziplinären Verständnisses nicht nur dem wissenschaftlichen Erkenntnisgewinn dienen, sondern auch praxisrelevante Resultate produzieren (vgl. Kromrey 2006: 16). In den Fallstudien wurden die Möglichkeiten und Grenzen der Instrumente des Dorferneuerungsprozesses bezüglich der Einstellungen und Handlungen der Akteure her- 
ausgearbeitet. Zusammenfassend lässt sich feststellen, dass im Rahmen des Dorferneuerungsverfahrens vielseitige Ansatzpunkte für eine Reduzierung der Flächeninanspruchnahme vorhanden sind. Mit der Dorferneuerung liegt also ein multi-instrumenteller Ansatz zur Bearbeitung des Themas Flächensparen vor. Doch es wurde auch deutlich, dass das bestehende Instrumentarium noch nicht systematisch ausgeschöpft wird. Im Sinne eines nachhaltigen Landmanagements sollte ein Monitoring bzw. eine Evaluierung in den Dorferneuerungsprozess eingeführt werden. Dabei sollte geprüft werden, welche Ziele in Maßnahmen einfließen und ob auch adäquate Maßnahmen für eine Reduzierung der Flächeninanspruchnahme ergriffen, umgesetzt und dauerhaft verankert wurden. So würde das abstrakte normative Ziel einen höheren Stellenwert bekommen und auch Maßnahmen nach sich ziehen. Hierfür ist eine Operationalisierung des Ziels nötig, die mit der Berechnung von Folgekosten der Flächenneuinanspruchnahme einhergehen könnte.

Die Zielsetzung, im Dorferneuerungsprozess die Flächeninanspruchnahme zu reduzieren, steht - das zeigen die Fallstudien deutlich - nicht im Vordergrund. In den regulativ-hoheitlichen Instrumenten (der Dorferneuerung und der Bauleitplanung) böte sich jedoch vielfach die Gelegenheit, das Thema stärker hervorzuheben. Zur Sicherung von Überlegungen zur Freihaltung von Flächen und zur Innenentwicklung sollte im Rahmen der Dorferneuerung noch gezielter mit der Gemeinde kooperiert werden, was den Einsatz hoheitlicher und formeller Instrumente betrifft. Ebenso kommt der Verbesserung innergemeindlicher und interkommunaler Abstimmungsprozesse in Bezug auf die Siedlungsentwicklung eine große Bedeutung zu. Vielfach werden Dorferneuerungsverfahren bereits in Gemeindeentwicklungskonzepte und Integrierte Ländliche Entwicklungskonzepte eingebunden. In diesem Rahmen sollte jedoch stärker auf das heikle Thema der Neuausweisung von Wohn- und Gewerbeflächen eingegangen werden, um sowohl zur Bewusstseinsbildung wie auch zur Verankerung in kommunalen Gremien im Sinne einer Selbstverpflichtung und zur Umsetzung von Strategien beizutragen.

Darüber hinaus sollte bei den zuständigen Akteuren (vor allem Mitarbeiter der Verwaltung für Ländliche Entwicklung, Vorstand der Teilnehmergemeinschaft, Gemeinderat) das Bewusstsein für die weiteren Potenzialitäten der Instrumente verbessert werden. Kernkompetenzen der Dorferneuerung liegen im Einsatz von Bodenordnung und der Einbindung der Grundeigentümer in Entscheidungsprozesse. Diese Stärken könnten noch viel umfassender zur Unterstützung der Innenentwicklung und der Reduzierung der Flächenneuinanspruchnahme genutzt werden, z. B. in Verbindung mit planerischen Instrumenten durch Bodenordnungs- und Rückbaukonzepte. Einige planerische und infor- matorische Möglichkeiten, wie beispielsweise die frühzeitige Erfassung von Leerständen und Baulücken durch den Vitalitäts-Check für Innenentwicklung mit integrierter Flächenmanagement-Datenbank, sind bekannt und kommen in der Praxis in mehreren Ämtern für Ländliche Entwicklung inzwischen regelmäßig zur Anwendung.

Offene Forschungsfragen ergeben sich insbesondere in Bezug auf die systematische Weiterentwicklung des Instrumentariums unter Verwendung inter- und transdisziplinärer Methoden. Ein zentrales Dilemma stellt häufig die Akzeptanz von Forschungsergebnissen in der Praxis dar. Neuere Ansätze legen daher Wert auf die Durchführung von Forschungen im inter- und transdisziplinären Austausch (vgl. Rogga 2015). Die Untersuchungen werden gleichberechtigt von Wissenschaftlern und Praktikern (z. B. Mitarbeitern der Verwaltung) durchgeführt, sodass die Relevanz der Forschung für die lebensweltliche Anwendung sichergestellt werden kann. Das untersuchte Thema der Reduzierung der Flächeninanspruchnahme ist ganz zentral durch die Frage geprägt, warum bestimmte (theoretische) Überlegungen in der Praxis nicht zur Anwendung kommen. Weiterführende Untersuchungen sollten anhand des Forschungsgegenstandes die Hemmnisse inter- und transdisziplinären Handelns weiter erforschen und praxisrelevante Ergebnisse für den Abbau von Kooperationshemmnissen sammeln. Das Forschungsdesign sollte dafür inter- und transdisziplinär angelegt sein. Forschungskonsortien, die unterschiedliche disziplinäre Hintergründe haben, bieten durch die Zusammenführung verschiedener theoretischer Annahmen und Methoden Erkenntnisgewinne für komplexe Probleme. Untypisch für ingenieurwissenschaftliche Forschungen, jedoch sehr vielversprechend wäre beispielsweise eine Zusammenführung mit sozialwissenschaftlichen und psychologischen Methoden (vgl. Ansätze bei Müller-Christ 2013 oder Levin-Keitel/Lelong/Thaler 2017 mit systemischen Aufstellungen; Wehrmann 2005 mit Psychodrama).

\section{Literatur}

Bayerische Staatsregierung (2013): Landesentwicklungsprogramm Bayern (LEP) vom 1. September 2013. München.

Bayerische Verwaltung für Ländliche Entwicklung (2014): Planen mit System. Vitalitäts-Check 2.0 zur Innenentwicklung für Dörfer und Gemeinden. Leitfaden. München.

Bayerischer Gemeindetag (2015): Reform des Landesentwicklungsprogramms. Gemeindetag sieht Staatsregierung auf richtigem Weg. Pressemitteilung vom 15. April 2015. München.

BBR - Bundesamt für Bauwesen und Raumordnung (Hrsg.) (2007): Perspektive Flächenkreislaufwirtschaft. Kreislaufwirtschaft in der städtischen/stadtregionalen Flächennutzung - Fläche im Kreis. Band 3: Neue Instrumente für neue Ziele. Bonn.

Blatter, J.; Janning, F.; Wagemann, C. (2007): Qualitative Politikanalyse. Eine Einführung in Forschungsansätze und Methoden. Wiesbaden. https://doi.org/10.1007/978-3-531-90716-1

BMVBS - Bundesministerium für Verkehr, Bau und Stadtentwicklung; BBSR - Bundesinstitut für Bau-, Stadt- und Raumforschung 
(Hrsg.) (2009): Einflussfaktoren der Neuinanspruchnahme von Flächen. Bonn. = Forschungen 139.

Bock, S.; Hinzen, A.; Libbe, J. (Hrsg.) (2011): Nachhaltiges Flächenmanagement. Ein Handbuch für die Praxis. Berlin.

Bock, S.; Preuß, T. (2011): Flächenverbrauch: Fakten, Trends und Ursachen. In: Bock, S.; Hinzen, A.; Libbe, J. (Hrsg.): Nachhaltiges Flächenmanagement. Ein Handbuch für die Praxis. Berlin, 21-31.

Boos-Krüger, A. (2009): Ortsübergreifende Innenentwicklung in der Hessischen Dorfentwicklung. Unveröffentlichtes Gutachten für das Hessische Ministerium für Umwelt, Energie, Landwirtschaft und Verbraucherschutz. Wiesbaden.

Braun, D. (2000): Politische Gesellschaftssteuerung zwischen System und Akteur. In: Lange, S.; Braun, D. (Hrsg.): Politische Steuerung zwischen System und Akteur. Eine Einführung. Wiesbaden, 99-176. = Grundwissen Politik 30. https://doi.org/10.1007/978-3663-11096-5

DASL - Landesgruppe Bayern der Deutschen Akademie für Städtebau und Landesplanung; Bayerischer Landesverein für Heimatpflege; BUND Naturschutz in Bayern; Bayerische Architektenkammer (2015): Heimat Bayern 2020 - Ausverkauf unserer bayerischen Kulturlandschaft? München.

Dehne, P. (2010): Dorferneuerung. In: Henckel, D.; von Kuczkowski, K.; Lau, P.; Pahl-Weber, E.; Stellmacher, F. (Hrsg.): Planen - Bauen - Umwelt. Ein Handbuch. Wiesbaden, 116-120.

Deutsche Bundesregierung (2002): Perspektiven für Deutschland. Unsere Strategie für eine nachhaltige Entwicklung. Langfassung. Berlin.

Diller, C. (2013): Ein nützliches Forschungswerkzeug! Zur Anwendung des Akteurzentrierten Institutionalismus in der Raumplanungsforschung und den Politikwissenschaften. In: PND online $1,1-15$.

Dosch, F. (2010): Flächenkreislaufwirtschaft als akteursorientiertes Modell der Siedlungsflächenentwicklung. In: Klemme, M.; Selle, K. (Hrsg.): Siedlungsflächen entwickeln. Akteure, Interdependenzen, Optionen. Detmold, 196-213.

Einig, K. (2010): Korporativer Akteur Kommune: Bedeutung baulandpolitischer Ziele und akteurszentrierter Verhaltensmodelle. In: Klemme, M.; Selle, K. (Hrsg.) (2010): Siedlungsflächen entwickeln. Akteure, Interdependenzen, Optionen. Detmold, 169195.

Fürst, D. (2010a): Akteure der Planung. In: In: Henckel, D.; von Kuczkowski, K.; Lau, P.; Pahl-Weber, E.; Stellmacher, F. (Hrsg.): Planen - Bauen - Umwelt. Ein Handbuch. Wiesbaden, 18-21.

Fürst, D. (2010b): Raumplanung. Herausforderungen des deutschen Institutionensystems. Detmold.

Fürst, D. (2015): Regional Governance oder ,des Kaisers neue Kleider"? - Stand der Diskussion. In: PND online 1, 1-15.

Fürst, D.; Scholles, F. (Hrsg.) (2008): Handbuch Theorien und Methoden der Raum- und Umweltplanung. Dortmund.

George, A. L.; Bennett, A. (2005): Case Studies and Theory Development in the Social Sciences. Cambridge.

Goetzke, R.; Schlump, C.; Hoymann, J.; Beckmann, G.; Dosch, F. (2014): Flächenverbrauch, Flächenpotenziale und Trends 2030. Bonn. = BBSR-Analysen KOMPAKT 7/2014.

Grube, J. (2006): Lebensraum Dorf. Methoden, Inhalte und Ergebnisse der Dorferneuerung. Berlin.

GTZ - Deutsche Gesellschaft für Technische Zusammenarbeit (o.J.): Mainstreaming Participation. Instrumente zur AkteursAnalyse. 10 Bausteine für die partizipative Gestaltung von Kooperationssystemen. Eschborn.

Hanns-Seidel-Stiftung (1989): Das Bayerische Dorferneuerungsprogramm. München. $=$ Kommunalpolitischer Leitfaden 9.

Henger, R.; Schier, M.; Voigtländer, K. (2015): Der künftige Bedarf an Wohnungen. Eine Analyse für Deutschland und alle 402 Kreise. Köln. = IW policy paper 24/2015.

Henkel, G. (2012): Das Dorf. Landleben in Deutschland - gestern und heute. Stuttgart.
Hirsch Hadorn, G.; Wölfing Kast, S. (2002): Optionen und Restriktionen - eine Heuristik für transdisziplinäre Nachhaltigkeitsforschung. In: Hirsch Hadorn, G.; Maier, S.; Wölfing Kast, S. (Hrsg.): Transdisziplinäre Forschung in Aktion. Optionen und Restriktionen nachhaltiger Ernährung. Zürich, 9-52.

Jering, A.; Lindemann, H.-H.; Seidel, W.; Musolff, A.; Burger, A.; Berg, H.; Wehrspaun, M.; Locher, B.; Hülsmann, W.; Solms, J.; Penn-Bressel, G.; Dickow-Hahn, R.; Roy, L.; Bunge, T.; Röthke, P.; Verron, H.; Huckestein, B.; Gohlisch, G.; Rechenberg, J.; Borowski, I.; Schmidt, S.; Giese, E.; Werner, B.; Grimski, D.; Kälberer, A.; Weiland-Wascher, A.; Henseling, K.-O. (2003): Reduzierung der Flächeninanspruchnahme durch Siedlung und Verkehr. Materialienband. Berlin. = UBA-Texte 90/2003.

Job, H.; Pütz, M. (Hrsg.) (2006): Flächenmanagement. Grundlagen für eine nachhaltige Siedlungsentwicklung mit Fallbeispielen aus Bayern. Hannover. = Arbeitsmaterial der ARL 322.

Jürges, N. (2016): Wahrnehmungen und Funktionen in der Transformation zur Bioökonomie - Eine Akteursanalyse im Politikfeld „Boden“. Leipzig. = UFZ Discussion Papers 6/2016.

Klemme, M. (2009): Stadtentwicklung ohne Wachstum. Zur Praxis kommunaler Siedlungsflächenentwicklung. Empirische Befunde und Folgerungen zu Steuerungsverständnissen und -formen öffentlicher Akteure. Dissertation an der Rheinisch-Westfälischen Technischen Hochschule Aachen.

Kötter, T.; Langer, J. (2014): Innenentwicklung der Dörfer in Brandenburg - komplexe Handlungsfelder und innovative Strategien. In: Flächenmanagement und Bodenordnung 76, 4, 156-165.

Kötter, T.; Voß, W. (2012): Dorfentwicklung, Dorferneuerung, Dorfumbau. In: Kummer, K.; Frankenberger, J. (Hrsg.): Das deutsche Vermessungs- und Geoinformationswesen 2013. Berlin, 221-260.

Kötter, T; Weigt, D.; Frielinghaus, B.; Schetke, S. (2009): Nachhaltige Siedlungs- und Flächenentwicklung. Inhaltliche und methodische Aspekte der Erfassung und Bewertung. In: Hepperle, E.; Lenk, H. (Hrsg.): Land Development Strategies: Patterns, Risks, and Responsibilities. Zürich, 61-83.

Kromrey, H. (2006): Empirische Sozialforschung. Modelle und Methoden der standardisierten Datenerhebung und Datenauswertung. Stuttgart.

Lamnek, S. (1995): Qualitative Sozialforschung. München.

Levin-Keitel, M.; Lelong, B.; Thaler, T. (2017): Zur Darstellung von Macht in der räumlichen Planung - Potenziale und Grenzen der Methode der systemischen Aufstellung. In: Raumforschung und Raumordnung I Spatial Research and Planning 75, 1, 31-44.

LfU - Bayerisches Landesamt für Umwelt (2009): Flächenmanagement in interkommunaler Zusammenarbeit. Endbericht. Augsburg.

Lintzmeyer, F.; Schwarz, C.; Müller-Herbers, S.; Fina, S. (2015): Verknüpfung von Innenentwicklung und Daseinsvorsorge in der Ländlichen Entwicklung - das Instrument Vitalitäts-Check 2.0. In: BBSR - Bundesinstitut für Bau-, Stadt- und Raumforschung (Hrsg.): Perspektiven der Regionalentwicklung in Schrumpfungsregionen. Bonn, 89-96. = BBSR-Online-Publikation 18/2015.

Magel, H. (1991): Dorferneuerung in Deutschland. Stuttgart.

Magel, H. (2014): Von der Flurbereinigung zum Landmanagement Lehre und Forschung für die Welt von morgen. In: Bayerischer Gemeindetag 12, 510-511.

Magel, H.; Franke, S. (2007): 21 Gemeinden - Gute Beispiele für eine nachhaltige Kommunalentwicklung. Endbericht zum Forschungsvorhaben im Auftrag des Bayerischen Staatsministeriums für Umwelt, Gesundheit und Verbraucherschutz. München.

Malburg-Graf, B.; Schmettow, P. (2012): Chefsache Innenentwicklung. Kollegiales Coaching zum Flächenmanagement unterstützt Bürgermeister ländlicher Gemeinden. In: Standort. Zeitschrift für Angewandte Geographie 36, 4, 187-193. https://doi.org/10.1007/ s00548-012-0236-y

Mayntz, R. (2005): Governance Theory als fortentwickelte Steuerungstheorie? In: Schuppert, G. F. (Hrsg.): Governance-For- 
schung. Vergewisserung über Stand und Entwicklungslinien. Baden-Baden, 11-20.

Menzl, M. (2014): Nimby-Proteste - Ausdruck neu erwachten Partizipationsinteresses oder eines zerfallenden Gemeinwesens? In: Gestring, N.; Ruhne, R.; Wehrheim, J. (Hrsg.): Stadt und soziale Bewegungen. Wiesbaden, 65-81. https://doi.org/10.1007/9783-658-01398-1_4

Meuser, M.; Nagel, U. (2005): ExpertInneninterviews - vielfach erprobt, wenig bedacht. Ein Beitrag zur qualitativen Methodendiskussion. In: Bogner, A.; Littig, B.; Menz, W. (Hrsg.): Das Experteninterview. Theorie, Methode, Anwendung. Opladen, 71-93.

Milbert, A. (2015): Wachsen oder schrumpfen? Bonn. = BBSR-Analysen KOMPAKT 12/2015.

Möckel, S. (2015): Politische und rechtliche Ziele zum vorsorgenden Schutz der Böden in Deutschland. In: Natur und Landschaft 90, 11, 497-502. https://doi.org/10.17433/11.2015.50153365.497502

Müller-Christ, G. (2013): Wo stehen Nachhaltigkeit und Ressourcenorientierung im Unternehmen? Ordnungsangebote im Prämissengerangel durch Systemaufstellungen. In: Klinke, S.; Rohn, H. (Hrsg.): RessourcenKultur. Vertrauenskulturen und Innovationen für Ressourceneffizienz im Spannungsfeld normativer Orientierung und betrieblicher Praxis. Baden-Baden, 383-392. = Nachhaltige Entwicklung 4.

Naturkapital Deutschland - The Economics of Ecosystems and Biodiversity (TEEB) (2012): Der Wert der Natur für Wirtschaft und Gesellschaft - Eine Einführung. München.

Paul A.; Taschner W. (2017): Fluch und Segen neuer Gewerbeflächen. In: Bayerische Staatszeitung vom 31. März 2017, 18.

Peter, H.; Fengler, B.; Moser, A. (2013): Welches Potenzial bietet die Dorferneuerungsförderung in Hessen, Mecklenburg-Vorpommern, Niedersachsen, Nordrhein-Westfalen und SchleswigHolstein zur Innenentwicklung von Dörfern? In: Raumforschung und Raumordnung 71, 1, 49-64. https://doi.org/10.1007/s13147012-0207-6

Ratter, B. M. W. (2006): Komplexitätstheorie und Geographie - Ein Beitrag zur Begründung einer anderen Sicht auf Systeme. In: Mitteilungen der Österreichischen Geographischen Gesellschaft 148, 109-124.

Rill, L.; Drago, B.; Groß, C. (2014): Vitalitäts-Check 2.0. Ein datenbankgestütztes Analyseinstrument zur Innenentwicklung für Dörfer, Gemeinden und interkommunale Kooperationen. In: DVWMitteilungen 66, 4, 403-411.

Ritzinger, A. (2016): Flächensparen zwischen Theorie und Praxis. Eine akteursorientierte Analyse des Dorferneuerungsprozesses. Dissertation an der Technischen Universität München.

Rogga, S. (2015): Transdisziplinarität - Grenzüberschreitung als Prinzip: Erfahrungen aus transdisziplinärer Forschung für ein Nachhaltiges Landmanagement. Müncheberg.

Scharpf, F. (2006): Interaktionsformen. Akteurzentrierter Institutionalismus in der Politikforschung. Wiesbaden.

Schiller, G.; Gutsche, J.-M.; Deilmann, C. (2009): Von der Außenzur Innenentwicklung in Städten und Gemeinden. Das Kostenparadoxon der Baulandentwicklung. Dessau-Roßlau. = UBA-Texte $31 / 2009$.

Schmalholz, M. (2005): Steuerung der Flächeninanspruchnahme. Defizite des Umwelt- und Planungsrechts sowie alternative Ansätze zur Reduzierung des Flächenverbrauchs durch Siedlung und Verkehr. Norderstedt.

Schmied, D. (2009): Interkommunale Allianz Oberes Werntal: Gemeinsam von der Außen- zur Innenentwicklung. In: Ländlicher Raum 60, 3, 18-21.
Schwing, R.; Fryszer, A. (2015): Systemisches Handwerk. Werkzeug für die Praxis. Göttingen.

Sebald, C. (2015): Zersiedelung. Hügel, Hallen und Hotels. In: Süddeutsche Zeitung vom 30. Juni 2015, 59.

Selle, K. (2005): Planen. Steuern. Entwickeln. Über den Beitrag öffentlicher Akteure zur Entwicklung von Stadt und Land. Dortmund.

Siedentop, S. (2010a): Innenentwicklung/Außenentwicklung. In: Henckel, D.; von Kuczkowski, K.; Lau, P.; Pahl-Weber, E.; Stellmacher, F. (Hrsg.): Planen - Bauen - Umwelt. Ein Handbuch. Wiesbaden, 235-240.

Siedentop, S. (2010b): Folgekosten der Siedlungsentwicklung als Wahrnehmungsproblem. In: Klemme, M.; Selle, K. (Hrsg.): Siedlungsflächen entwickeln. Akteure, Interdependenzen, Optionen. Detmold, 153-168.

Spangenberger, V. (2008): Raumordnerische Rahmenbedingungen und Vorgaben für den Stadt- und Dorfumbau. In: Flächenmanagement und Bodenordnung 70, 2, 49-55.

SRL - Vereinigung für Stadt-, Regional- und Landesplanung; bdla Bund Deutscher Landschaftsarchitekten (2017): Stellungnahme der SRL und des bdla zur Einführung des § 13b BauGB. Berlin.

Steffenhagen, P.; Weitkamp, A. (2015): Perspektive „Leerstand“ im Dorf: Möglichkeiten zum Einsatz zivilgesellschaftlichen Engagements. In: Neues Archiv für Niedersachsen 1, 70-81.

StMELF - Bayerisches Staatsministerium für Ernährung, Landwirtschaft und Forsten (2012): Ländliche Entwicklung in Bayern. München.

StMELF - Bayerisches Staatsministerium für Ernährung, Landwirtschaft und Forsten (2015): Dorferneuerungsrichtlinien (DorfR 2015) zum Vollzug des Bayerischen Dorfentwicklungsprogramms. Bekanntmachung vom 22. Dezember 2014. München.

StMLF - Bayerisches Staatsministerium für Landwirtschaft und Forsten (2006): Aktionsprogramm Dorf vital. Innenentwicklung in der Dorferneuerung. München. $=$ Materialien zur ländlichen Entwicklung 40.

Ulmer, F.; Renn, O.; Ruther-Mehlis, A.; Jany, A.; Lilienthal, M.; Malburg-Graf, B.; Pietsch, J.; Selinger, J. (2007): Erfolgsfaktoren zur Reduzierung des Flächenverbrauchs in Deutschland. Eine Studie im Auftrag des Rates für nachhaltige Entwicklung. Berlin.

Vereinte Nationen (2013): Resolution der Generalversammlung A/Res/ 68/232. Weltbodentag und Internationales Jahr der Böden. New York.

Voß, W.; Güldenberg, E.; Kirsch-Stracke, R.; Streibel, N. (2011): Dörfer im Schrumpfungsprozess - Handlungsempfehlungen für die Dorfentwicklung und den Umgang mit Gebäudeleerständen. In: Flächenmanagement und Bodenordnung 73, 4, 165-171.

Wehrmann, B. (2005): Landkonflikte im urbanen und peri-urbanen Raum von Großstädten in Entwicklungsländern. Münster. $=$ Forschungsbeiträge zur Stadt- und Regionalgeographie 2.

Weith, T.; Besendörfer, C.; Gaasch, N.; Kaiser, D. B.; Müller, K.; Repp, A.; Rogga, S.; Strauß, C.; Zscheischler, J. (Hrsg.) (2013): Nachhaltiges Landmanagement: Was ist das? Müncheberg. = LeibnizZentrum für Agrarlandschaftsforschung, Diskussionspapier 7.

Wiechmann, T. (2014): Planung ohne Plan: Der Ausnahmezustand als Regelfall innovativer Raumentwicklung. In: Hohn, U.; Kemming, H.; Reimer, M. (Hrsg.): Formate der Innovation in der Stadt- und Regionalentwicklung. Detmold, 21-42. = Metropolis und Region 13.

Wilz, S. M. (2010): Entscheidungsprozesse in Organisationen. Eine Einführung. Wiesbaden. 